\title{
Relación entre las creencias disfuncionales y estímulos de alto riesgo con el craving en pacientes consumidores de pasta básica de cocaína
}

\author{
Relationship between dysfunctional beliefs and \\ Incentives high risk patients with craving in basic pasta \\ consumers of cocaine
}

Recibido: 09/07/2015 Revisado: 21/07/2015 Aceptado: 15/09/2015

Gustavo Augusto Sipán Valerio

\section{RESUMEN}

Objetivo: Determinar la relación que existe entre las creencias disfuncionales y estímulos de alto riesgo con el craving en pacientes consumidores de pasta básica de cocaína del Departamento de Adicciones del Hospital Víctor Larco Herrera de Lima de enero a julio del 2014. Materiales y métodos: Estudio cuantitativo correlacional, transversal y retrospectivo. Se seleccionó una muestra de 50 pacientes consumidores de pasta básica de cocaína (PBC) determinada según el muestreo aleatorio simple, con variable continua. Se aplicó un muestreo piloto de tamaño, $\mathrm{n}$ $=15$, con el fin de probar su funcionalidad, el cual se sometió a un análisis de confiabilidad y validez. La técnica empleada fue la encuesta, a través de 03 instrumentos, para la construcción y clarificación de las 3 variables se utilizó la escala de estaninos la cual tomó en cuenta los puntajes y su clasificación fue ordinal. La información fue codificada y digitada en Excel y exportada al software estadístico SPSS, se utilizó la estadística descriptiva y la estadística inferencial para demostrar la relación entre las variables haciendo uso del test no paramétrico chi cuadrado. Resultados: Que la relación entre las creencias nucleares $\left(X^{2}=17,398, p=0,002\right)$, las creencias adictivas $\left(X^{2}=9,678\right.$, $p=0,046)$, las creencias anticipadoras $\left(X^{2}=11,916\right.$, $p=0,018)$, las creencias permisivas $\left(X^{2}=9,306, p=0,054\right)$, los estímulos externos $\left(X^{2}=14,236, p=0,007\right)$ y los estímulos internos $\left(X^{2}=10,485, p=0,033\right)$, con el craving en pacientes consumidores de PBC; es significativa. Conclusiones: La relación entre las creencias disfuncionales y estímulos de alto riesgo con el craving en pacientes consumidores de PBC del Departamento de Adicciones del Hospital Víctor Larco Herrera de Lima es significativa

Palabras clave: Creencias disfuncionales, craving, estímulos de alto riesgo, pacientes consumidores de pasta básica de cocaína.

\section{ABSTRACT}

Objective: To determine the relationship between dysfunctional beliefs and stimuli with high risk patients into consumers craving cocaine base Department of Addiction Victor Larco Herrera Lima Hospital from January to July
2014. Materials and methods: correlational, retrospective longitudinal quantitative study. A sample of 50 patients with cocaine paste consumers determined by simple random sampling with continuous variable was selected. And a pilot size, $n=15$ sample, in order to steal their functionality, which was subjected to reliability and validity analysis was applied. The technique used was the Survey, through 03 instruments for construction and clarification of the 3 variables statins scale which took into account the scores and ordinal classification was used. The information was coded and typed in Excel and exported to the statistical software SPSS, descriptive statistics and inferential statistics were used to demonstrate the relationship between the variables using the nonparametric chi-square test. Results: What core beliefs $\left(X^{2}=17.398, p=0.002\right)$ and addictive beliefs $\left(X^{2}=9.678, \quad p=0.046\right)$, anticipatory beliefs $\left(X^{2}=11.916\right.$, $p=0.018)$, permissive beliefs $\left(X^{2}=9.306, p=0.054\right)$, external stimuli $\left(X^{2}=14.236, p=0.007\right)$ and internal stimuli $\left(X^{2}=10.485, p=0.033\right)$, the relationship is significant with the craving for crack users. Conclusions: It is concluded that significant selection between dysfunctional beliefs and stimuli with high-risk consumers craving in patients cocaine paste Department of Addiction Hospital Victor Larco Herrera in Lima.

Keywords: Dysfunctional beliefs, craving, stimulation of high-risk consumers $\mathrm{PBC}$ patients.

\section{INTRODUCCIÓN}

El consumo de drogas a nivel mundial es un fenómeno de múltiples causas, que impacta de diferentes maneras en la población en general y atenta contra la salud pública, el desarrollo social y familiar de las personas, afectando incluso la seguridad individual y colectiva. En nuestro país, de acuerdo con la IV Encuesta Nacional de Consumo de Drogas en la Población General del Perú 2010 realizada por Devida (2011), se tiene que el $5,1 \%$ de la población ha hecho uso de drogas ilegales en algún momento de su vida. El número de consumidores en el último año para todas las drogas ilegales asciende a 264,121 , siendo la marihuana $(168,245)$ y la pasta básica de cocaína $(75,506)$ las más usadas. (INPE y Devida, 2013). 
Las drogas sociales siguen siendo las de mayor consumo a nivel nacional y en la adolescencia el uso experimental de drogas ilegales se vuelve cada día más común en las escuelas y barrios. Nuevas amenazas están apareciendo, incluyendo el éxtasis y las drogas de síntesis, asociadas a contextos de diversión, ampliando así el panorama del problema de las drogas en el Perú. A lo largo de los años mucho se ha intentado hacer para enfrentar este flagelo. Desde iniciativas de penalización y castigo, hasta propuesta de legalización que no han hecho más que incentivar la discusión. En este camino lo único que ha quedado claro es que el afronte debe ser multisectorial y que no puede promoverse una visión aislada de la problemática. Los países deben necesariamente comprometerse en la lucha contra las drogas pero considerando que esta responsabilidad no debe recaer únicamente en las autoridades sino que es un tema de todos los sectores, el público y el privado; las familias y las organizaciones los más gravemente afectados al igual que aquellos que ven el problema de las drogas como algo lejano.

Se requiere un abordaje integral en lo preventivo y remediativo, con especial énfasis en los sectores de riesgo; reconociendo que una meta básica es evitar que más personas se inicien en el consumo, que quienes ya han ingresado en adicción encuentren reales posibilidades de tratamiento y que los que han logrado sobreponerse al consumo alcancen mejores formas de reinserción social. (Cedro, 2011). Con respecto a los individuos consumidores de pasta básica de cocaína (PBC), el cual llegan a las instituciones especializadas para su tratamiento y rehabilitación, se generan una serie de aspectos tanto en el individuo consumidor y en su contexto que el equipo terapéutico debe tener en cuenta para proyectar mejores probabilidades de recuperación. Dentro de este marco debemos mencionar las creencias adictivas como formas que las personas utilizan para interpretar determinadas situaciones que influyen en sus sentimientos, motivaciones y acciones (Aaron Beck, et al, 2013).

Algunas creencias específicas como estas constituyen una vulnerabilidad al abuso de sustancias. Cuando se activan ante circunstancias determinadas predecibles, las creencias incrementan la probabilidad del uso continuado de droga o alcohol (es decir, estimulan el craving). Es típico que las creencias sobre la utilización de las drogas se activen en circunstancias específicas, que suelen llamarse situaciones estímulo. Sin embargo, dependiendo del estado de ánimo del paciente en ese momento y de su autocontrol, el grado de riesgo de una situación variará considerablemente de un momento a otro. Estas circunstancias, que pueden ser tanto externas como internas, corresponden a lo que Marlatt y Gordon (1985) denominaron situaciones de alto riesgo. Estas situaciones estimulan el craving de fumar, esnifar o ingerir drogas.

Los craving y los impulsos irrefrenables por consumir, son los factores más importantes de abandono terapéutico e incluso a veces llegan a provocar la recaída después de largos períodos de abstinencia, sobre todo porque resurgen durante y después del tratamiento (Annis, 1986; Caroll, Rounsaville y Keller, 1991; Childress, et al, 1990; Covi, et al, 1990; Horvath, 1988, en prensa; Shulman, 1989; Tiffany, 1990; Washton, 1988). (Aaron Beck, et al, 2013; pág. 215). Por tanto estos aspectos de los pacientes consumidores de $\mathrm{PBC}$ que se encuentran en tratamiento en el Departamento de Adicciones, del Hospital Víctor Larco Herrera, han generado un gran interés para la investigación científica y con los resultados proporcionar nuevos contextos que induzcan a mejorar la perspectiva del abordaje psicoterapéutico en ellos.

La investigación tiene como objetivo general determinar la relación que existe entre las creencias disfuncionales y estímulos de alto riesgo con el craving en pacientes consumidores de pasta básica de cocaína del departamento de adicciones del Hospital Víctor Larco Herrera. Dentro de los objetivos específicos tenemos: Establecer la relación entre las creencias nucleares, creencias adictivas, creencias anticipadoras, creencias permisivas y estímulos de alto riesgo internos y externos con el craving de la población objeto de estudio.

\section{MATERIALES Y MÉTODOS}

En el presente estudio según el problema y los objetivos planteados, el tipo de investigación es correlacional ya que miden las relaciones que puede o no darse entre las variables, creencias disfuncionales y estímulos de alto riesgo con el craving en pacientes consumidores de PBC. Además es de enfoque cuantitativo porque usa la recolección de datos para probar hipótesis, con base en la medición numérica y el análisis estadístico, para establecer patrones de comportamiento y probar teorías.

Asimismo es de un diseño no experimental porque permite explorar fenómenos, describir la ocurrencia, su magnitud, diversas características y afecciones; sin la manipulación deliberada de las variables, no se tiene control directo sobre dichas variables ni se puede influir sobre ellas, porque ya sucedieron, al igual que sus efectos. Es de corte transversal, porque las variables involucradas se miden en una sola oportunidad y de inmediato se procede a su descripción y análisis. Es un estudio reprospectivo, porque el investigador registrará los hechos que vivenciaron los pacientes consumidores de PBC.

\section{Muestra}

La población objeto de estudio, estuvo representada por el número de pacientes atendidos en el Departamento de Adicciones del Hospital Víctor Larco Herrera, de enero a mayo del 2014 , que fueron 78 pacientes de sexo masculino.

La determinación del tamaño de la muestra fue según el muestreo aleatorio simple, con variable continua, el cual después del procesamiento estadístico se tomó una muestra de $n=50$ pacientes.

\section{Técnica e instrumentos de recolección de datos}

En relación a los instrumentos de medición (IM) se aplicó un muestreo piloto de tamaño, $n=15$ pacientes de adicciones (consumidores de PBC), con el fin de probar su funcionalidad. Seguidamente los IM se sometieron a un análisis de confiabilidad y validez con la información obtenida en el muestreo piloto. Para las 03 variables del estudio: Craving, creencias disfuncionales y estímulos de alto riesgo se utilizaron los siguientes coeficientes de confiabilidad (CC): CC de las mitades según Spearman Brown y CC de las mitades según Browny, CC de las mitades según Rulon - Guttman y el CC Alfa de Cronbach, el cual dieron como resultado: Confiables. Asimismo también para la validez de los instrumentos fueron sometidos a evaluación de 07 jueces expertos en la especialidad y su validez fue sometida a través del coeficiente de Concordancia R de Finn, que en el transcurso del tiempo y la práctica resultó más confiable y preciso. 
La técnica empleada fue la encuesta, a través de los tres instrumentos que a continuación se detallan:

- Instrumento escala de Likert modificada para medir actitud y creencias sobre el craving en consumo de PBC que consta de 21 ítems.

Que tiene como base el cuestionario de creencias sobre el craving (CBQ), que fue desarrollada por Freed D. Wright. Es un autoinforme que mide las actitudes y creencias acerca del fenómeno asociado con el craving por consumir PBC. Cada uno de los 21 ítems se evaluó en una escala de 1 a 3 (p. ej., 1 = en desacuerdo, 2 = indiferente y $3=$ de acuerdo).

- Cuestionario de creencias disfuncionales acerca del abuso de pasta básica de cocaína que consta de 33 ítems.

Que tiene como base las creencias acerca de la utilización de sustancias, fue desarrollado por Freed D. Wright.

Es un autoinforme que mide las creencias disfuncionales más frecuentes acerca de la utilización de las drogas. Cada uno de los 33 items se evaluó en una escala que va de 1 a 5 (p. ej., 1 = totalmente en desacuerdo, 2 = en desacuerdo, 3 = indiferente, $4=$ de acuerdo y $5=$ totalmente de acuerdo).

- Instrumento escala de Likert modificada para medir actitud sobre estímulos de alto riesgo para consumo de $\mathrm{PBC}$ que consta de 23 ítems.

Este instrumento fue construido por el autor de la presente investigación, es un autoinforme que mide la actitud sobre los estímulos de alto riesgo para consumo de PBC. Cada uno de los 23 ítems se evaluó en una escala de 1 a 3 (p. ej., 1 = en desacuerdo, $2=$ indiferentes y $3=$ de acuerdo).

Para la construcción y clasificación de las 3 variables se utilizó la escala de estaninos la cual tomó en cuenta los puntajes de los pacientes y su clasificación fue ordinal.

Estos 03 instrumentos se aplicaron en horas libres de los pacientes, encuestándolos gradualmente hasta completar con los 50 usuarios.

La información previamente codificada fue digitada en Excel y exportada al software estadístico SPSS para su proceso y análisis de la data, se utilizó la estadística descriptiva para las variables en estudio y la estadística inferencial para demostrar la relación entre las mismas haciendo uso del test no paramétrico chi cuadrado. La información está presentada en cuadros de una o doble entrada con sus respectivos gráficos.

\section{RESULTADOS}

Un total de 50 pacientes consumidores de PBC del Departamento de Adicciones del Hospital Víctor Larco Herrera de la ciudad de Lima, participaron del estudio, todos varones, sus edades estuvieron comprendidas entre 19 y 50 años. El estudio indica que las creencias disfuncionales; como las creencias nucleares, creencias adictivas, creencias anticipadoras y creencias permisivas es significativo con el craving, es decir existe relación. Asimismo los estímulos de alto riesgo, externos e internos es significativo con el craving, es decir existe relación.

Tabla 1. Tabla de contingencia de creencias nucleares y actitud sobre el craving en pacientes consumidores de PBC del Hospital Víctor Larco Herrera. Lima - 2014.

\begin{tabular}{|c|c|c|c|c|c|}
\hline \multicolumn{2}{|c|}{ Creencias nucleares } & \multicolumn{3}{|c|}{ Craving en consumo de PBC } & \multirow{2}{*}{ Total } \\
\hline & & Acuerdo & Indiferente & Desacuerdo & \\
\hline Acuerdo & $\begin{array}{l}\mathrm{n} \\
\%\end{array}$ & $\begin{array}{c}9 \\
18,0 \%\end{array}$ & $\begin{array}{l}2 \\
4,0 \%\end{array}$ & $\begin{array}{l}1 \\
2,0 \%\end{array}$ & $\begin{array}{l}12 \\
24,0 \%\end{array}$ \\
\hline Indiferente & $\begin{array}{l}\mathrm{n} \\
\%\end{array}$ & $\begin{array}{c}7 \\
14,0 \%\end{array}$ & $\begin{array}{l}15 \\
30,0 \%\end{array}$ & $\begin{array}{c}5 \\
10,0 \%\end{array}$ & $\begin{array}{l}27 \\
54,0 \%\end{array}$ \\
\hline Desacuerdo & $\begin{array}{l}\mathrm{n} \\
\%\end{array}$ & $\begin{array}{l}1 \\
2,0 \%\end{array}$ & $\begin{array}{l}4 \\
8,0 \%\end{array}$ & $\begin{array}{c}6 \\
12,0 \%\end{array}$ & $\begin{array}{c}11 \\
22,0 \%\end{array}$ \\
\hline Total & $\begin{array}{l}\mathrm{n} \\
\%\end{array}$ & $\begin{array}{l}17 \\
34,0 \%\end{array}$ & $\begin{array}{l}21 \\
42,0 \%\end{array}$ & $\begin{array}{l}12 \\
24,0 \%\end{array}$ & $\begin{array}{c}50 \\
100,0 \%\end{array}$ \\
\hline
\end{tabular}

Fuente: Encuesta realizada a los pacientes del Departamento de Adicciones del HVLH. Enero - julio 2014.

Chi cuadrado $=17,398, \mathrm{gl}=4, \mathrm{p}=0,002$ Significativo

\section{Análisis e interpretación de los datos}

Las frecuencias observadas se sometieron a la siguiente prueba de hipótesis:

Hipótesis

$H_{\circ}$ : No existe relación entre las creencias nucleares y el craving en pacientes consumidores de PBC.

$\mathbf{H}_{1}$ : Existe relación entre las creencias nucleares y el craving en pacientes consumidores de PBC.

Nivel de significancia: Error tipo I, $\alpha=0,05$ (5\%). Esto es, el error que se cometería al rechazar la Ho, siendo esta verdadera.

Prueba Estadística: Prueba no paramétrica Chi cuadrado. Decisión Estadística: El Chi cuadrado observado es $\mathrm{Ji}^{2}=17,398$ con $\mathrm{gl}=4$, al cual le está asociado un valor de probabilidad, $P=0,002$. Puesto que este valor $P$ es menor 0 igual que $\alpha$, se concluye en rechazar la Ho. Es decir existe relación entre las creencias nucleares y el craving en pacientes consumidores de PBC. 
Tabla 2. Tabla de contingencia de creencias adictivas y actitud sobre el craving en pacientes consumidores de PBC del Hospital Víctor Larco Herrera. Lima - 2014.

\begin{tabular}{|c|c|c|c|c|c|}
\hline \multicolumn{2}{|c|}{ Creencias adictivas } & \multicolumn{3}{|c|}{ Craving en consumo de PBC } & \multirow[t]{2}{*}{ Total } \\
\hline & & Acuerdo & Indiferente & Desacuerdo & \\
\hline \multirow[t]{2}{*}{ Acuerdo } & $\mathrm{n}$ & 10 & 5 & 3 & 18 \\
\hline & $\%$ & $20,0 \%$ & $10,0 \%$ & $6,0 \%$ & $36,0 \%$ \\
\hline \multirow[t]{2}{*}{ Indiferente } & $\mathrm{n}$ & 6 & 12 & 4 & 22 \\
\hline & $\%$ & $12,0 \%$ & $24,0 \%$ & $8,0 \%$ & $44,0 \%$ \\
\hline \multirow[t]{2}{*}{ Desacuerdo } & $\mathrm{n}$ & 1 & 4 & 5 & 10 \\
\hline & $\%$ & $2,0 \%$ & $8,0 \%$ & $10,0 \%$ & $20,0 \%$ \\
\hline \multirow[t]{2}{*}{ Total } & $\mathrm{n}$ & 17 & 21 & 12 & 50 \\
\hline & $\%$ & $34,0 \%$ & $42,0 \%$ & $24,0 \%$ & $100,0 \%$ \\
\hline
\end{tabular}

Fuente: Encuesta realizada a los pacientes del Departamento de Adicciones del HVLH. Enero - julio 2014.

Chi cuadrado $=9,678, g l=4, p=0,046$ Significativo

Análisis e interpretación de los datos

Las frecuencias observadas se sometieron a la siguiente prueba de hipótesis:

Hipótesis

$H_{0}$ : No existe relación entre las creencias adictivas y el craving en pacientes consumidores de PBC.

$\mathrm{H}_{1}$ : Existe relación entre las creencias adictivas y el craving en pacientes consumidores de PBC .

Nivel de significancia: Error tipo I, $\alpha=0,05$ (5\%). Esto es, el error que se cometería al rechazar la Ho, siendo esta verdadera.

Prueba Estadística: Prueba no paramétrica Chi cuadrado. Decisión Estadística: El Chi cuadrado observado es $\mathrm{Ji}^{2}=9,678$ con $\mathrm{gl}=4$, al cual le está asociado un valor de probabilidad, $P=0,046$. Puesto que este valor $P$ es menor o igual que $\alpha$, se concluye en rechazar la Ho. Es decir existe relación entre las creencias adictivas y el craving en pacientes consumidores de PBC.

Tabla 3. Tabla de contingencia de creencias anticipadoras y actitud sobre el craving en pacientes consumidores de PBC del Hospital Víctor Larco Herrera. Lima - 2014.

\begin{tabular}{|c|c|c|c|c|c|}
\hline \multicolumn{2}{|c|}{ Creencias anticipadoras } & \multicolumn{3}{|c|}{ Craving en consumo de PBC } & \multirow[t]{2}{*}{ Total } \\
\hline & & Acuerdo & Indiferente & Desacuerdo & \\
\hline \multirow[t]{2}{*}{ Acuerdo } & $\mathrm{n}$ & 5 & 6 & 7 & 18 \\
\hline & $\%$ & $10,0 \%$ & $12,0 \%$ & $14,0 \%$ & $36,0 \%$ \\
\hline \multirow[t]{2}{*}{ Indiferente } & $\mathrm{n}$ & 10 & 11 & 0 & 21 \\
\hline & $\%$ & $20,0 \%$ & $22,0 \%$ &, $0 \%$ & $42,0 \%$ \\
\hline \multirow[t]{2}{*}{ Desacuerdo } & $\mathrm{n}$ & 2 & 4 & 5 & 11 \\
\hline & $\%$ & $4,0 \%$ & $8,0 \%$ & $10,0 \%$ & $22,0 \%$ \\
\hline \multirow[t]{2}{*}{ Total } & $\mathrm{n}$ & 17 & 21 & 12 & 50 \\
\hline & $\%$ & $34,0 \%$ & $42,0 \%$ & $24,0 \%$ & $100,0 \%$ \\
\hline
\end{tabular}

Fuente: Encuesta realizada a los pacientes del Departamento de Adicciones del HVLH. Enero - julio 2014.

$$
\text { Chi cuadrado }=11,916, \mathrm{gl}=4, \mathrm{p}=0,018 \text { Significativo }
$$

Análisis e interpretación de los datos

Las frecuencias observadas se sometieron a la siguiente prueba de hipótesis:

Hipótesis

$H_{\circ}$ : No existe relación entre las creencias anticipadoras y el craving en pacientes consumidores de PBC.

$\mathbf{H}_{1}$ : Existe relación entre las creencias anticipadoras y el
Craving en pacientes consumidores de PBC . Nivel de significancia: Error tipo I, $\alpha=0,05$ (5\%). Esto es, el error que se cometería al rechazar la Ho, siendo esta verdadera.

Prueba Estadística: Prueba no paramétrica Chi cuadrado. Decisión Estadística: El Chi cuadrado observado es $\mathrm{Ji}^{2}=11,916$ con $\mathrm{gl}=4$, al cual le está asociado un valor de 
probabilidad, $P=0,018$. Puesto que este valor $P$ es menor o igual que $\alpha$, se concluye en rechazar la Ho. Es decir existe relación entre las creencias anticipadoras y el craving en pacientes consumidores de PBC.

Tabla 4. Tabla de contingencia de creencias permisivas y actitud sobre el craving en pacientes consumidores de PBC del Hospital Víctor Larco Herrera. Lima - 2014.

\begin{tabular}{|c|c|c|c|c|c|}
\hline \multicolumn{2}{|c|}{ Creencias permisivas } & \multicolumn{3}{|c|}{ Craving en consumo de PBC } & \multirow[t]{2}{*}{ Total } \\
\hline & & Acuerdo & Indiferente & Desacuerdo & \\
\hline \multirow[t]{2}{*}{ Acuerdo } & $\mathrm{n}$ & 3 & 5 & 5 & 13 \\
\hline & $\%$ & $6,0 \%$ & $10,0 \%$ & $10,0 \%$ & $26,0 \%$ \\
\hline \multirow[t]{2}{*}{ Indiferente } & $\mathrm{n}$ & 13 & 14 & 3 & 30 \\
\hline & $\%$ & $26,0 \%$ & $28,0 \%$ & $6,0 \%$ & $60,0 \%$ \\
\hline \multirow[t]{2}{*}{ Desacuerdo } & $\mathrm{n}$ & 1 & 2 & 4 & 7 \\
\hline & $\%$ & $2,0 \%$ & $4,0 \%$ & $8,0 \%$ & $14,0 \%$ \\
\hline \multirow[t]{2}{*}{ Total } & $\mathrm{n}$ & 17 & 21 & 12 & 50 \\
\hline & $\%$ & $34,0 \%$ & $42,0 \%$ & $24,0 \%$ & $100,0 \%$ \\
\hline
\end{tabular}

Fuente: Encuesta realizada a los pacientes del Departamento de Adicciones del HVLH. Enero - julio 2014.

Chi cuadrado $=9,306, \mathrm{gl}=4, \mathrm{p}=0,054$ Significativo

Análisis e interpretación de los datos

Las frecuencias observadas se sometieron a la siguiente prueba de hipótesis:

Hipótesis

$\mathrm{H}_{0}$ : No existe relación entre las creencias permisivas y el craving en pacientes consumidores de PBC.

$\mathrm{H}_{1}$ : Existe relación entre las creencias permisivas y el craving en pacientes consumidores de PBC.

Nivel de significancia: Error tipo I, $\alpha=0,05$ (5\%). Esto es, el error que se cometería al rechazar la Ho, siendo esta verdadera.

Prueba Estadística: Prueba no paramétrica Chi cuadrado. Decisión Estadística: El Chi cuadrado observado es $\mathrm{Ji}^{2}=9.306$ con $\mathrm{gl}=4$, al cual le está asociado un valor de probabilidad, $P=0,054$. Puesto que este valor $P$ es menor o igual que $\alpha$, se concluye en rechazar la Ho. Es decir existe relación entre las creencias permisivas y el craving en pacientes consumidores de PBC.

Tabla 5. Tabla de contingencia de los estímulos externos de alto riesgo y actitud sobre el craving en pacientes consumidores de PBC del Hospital Víctor Larco Herrera. Lima - 2014.

\begin{tabular}{|c|c|c|c|c|c|}
\hline \multicolumn{2}{|c|}{ Estímulos externos } & \multicolumn{3}{|c|}{ Craving en consumo de PBC } & \multirow{2}{*}{ Total } \\
\hline & & \multirow{2}{*}{$\begin{array}{c}\text { Acuerdo } \\
10\end{array}$} & \multirow{2}{*}{$\frac{\text { Indiferente }}{5}$} & \multirow{2}{*}{$\begin{array}{c}\text { Desacuerdo } \\
7\end{array}$} & \\
\hline Acuerdo & $\mathrm{n}$ & & & & 22 \\
\hline & $\%$ & $20,0 \%$ & $10,0 \%$ & $14,0 \%$ & $44,0 \%$ \\
\hline \multirow[t]{2}{*}{ Indiferente } & $\mathrm{n}$ & 5 & 5 & 5 & 15 \\
\hline & $\%$ & $10,0 \%$ & $10,0 \%$ & $10,0 \%$ & $30,0 \%$ \\
\hline \multirow{2}{*}{ Desacuerdo } & $\mathrm{n}$ & 2 & 11 & 0 & 13 \\
\hline & $\%$ & $4,0 \%$ & $22,0 \%$ &, $0 \%$ & $26,0 \%$ \\
\hline \multirow[t]{2}{*}{ Total } & $\mathrm{n}$ & 17 & 21 & 12 & 50 \\
\hline & $\%$ & $34,0 \%$ & $42,0 \%$ & $24,0 \%$ & $100,0 \%$ \\
\hline
\end{tabular}

Fuente: Encuesta realizada a los pacientes del Departamento de Adicciones del HVLH. Enero - julio 2014.

$$
\text { Chi cuadrado }=14,236, \mathrm{gl}=4, \mathrm{p}=0,007 \text { Significativo }
$$


Análisis e interpretación de los datos

Las frecuencias observadas se sometieron a la siguiente prueba de hipótesis:

Hipótesis

$\mathbf{H}_{\mathrm{o}}$ : No existe relación entre los estímulos externos de alto riesgo y el craving en pacientes consumidores de PBC.

$\mathbf{H}_{1}$ : Existe relación entre los estímulos externos de alto riesgo y el craving en pacientes consumidores de PBC .

Nivel de significancia: Error tipo I, $\alpha=0,05$ (5\%). Esto es, el error que se cometería al rechazar la Ho, siendo esta verdadera.

Prueba Estadística: Prueba no paramétrica Chi cuadrado. Decisión Estadística: El Chi cuadrado observado es $\mathrm{Ji}^{2}=14,236$ con $\mathrm{gl}=4$, al cual le está asociado un valor de probabilidad, $P=0,007$. Puesto que este valor $P$ es menor o igual que $\alpha$, se concluye en rechazar la Ho. Es decir existe relación entre los estímulos externos de alto riesgo y el craving en pacientes consumidores de PBC.

Tabla 6. Tabla de contingencia de los estímulos internos de alto riesgo y actitud sobre el craving en pacientes consumidores de PBC del Hospital Víctor Larco Herrera. Lima - 2014.

\begin{tabular}{|c|c|c|c|c|c|}
\hline \multicolumn{2}{|c|}{ Estímulos internos } & \multicolumn{3}{|c|}{ Craving en consumo de PBC } & \multirow[t]{2}{*}{ Total } \\
\hline & & Acuerdo & Indiferente & Desacuerdo & \\
\hline Acuerdo & $\begin{array}{l}\mathrm{n} \\
\%\end{array}$ & $\begin{array}{c}10 \\
20,0 \%\end{array}$ & $\begin{array}{l}4 \\
8,0 \%\end{array}$ & $\begin{array}{l}2 \\
4,0 \%\end{array}$ & $\begin{array}{c}16 \\
32,0 \%\end{array}$ \\
\hline Indiferente & $\begin{array}{l}\mathrm{n} \\
\%\end{array}$ & $\begin{array}{c}6 \\
12,0 \%\end{array}$ & $\begin{array}{c}9 \\
18,0 \%\end{array}$ & $\begin{array}{c}5 \\
10,0 \%\end{array}$ & $\begin{array}{l}20 \\
40,0 \%\end{array}$ \\
\hline Desacuerdo & $\begin{array}{l}\mathrm{n} \\
\%\end{array}$ & $\begin{array}{l}1 \\
2,0 \%\end{array}$ & $\begin{array}{c}8 \\
16,0 \%\end{array}$ & $\begin{array}{c}5 \\
10,0 \%\end{array}$ & $\begin{array}{c}14 \\
28,0 \%\end{array}$ \\
\hline Total & $\begin{array}{l}\mathrm{n} \\
\%\end{array}$ & $\begin{array}{l}17 \\
34,0 \%\end{array}$ & $\begin{array}{l}21 \\
42,0 \%\end{array}$ & $\begin{array}{l}12 \\
24,0 \%\end{array}$ & $\begin{array}{c}50 \\
100,0 \%\end{array}$ \\
\hline
\end{tabular}

Fuente: Encuesta realizada a los pacientes del Departamento de Adicciones del HVLH. Enero - julio 2014.

$$
\text { Chi cuadrado }=10,485, \mathrm{gl}=4, \mathrm{p}=0,033 \text { Significativo }
$$

Análisis e interpretación de los datos

Las frecuencias observadas se sometieron a la siguiente prueba de hipótesis:

Hipótesis

$\mathbf{H}_{\mathrm{o}}$ : No existe relación entre los estímulos internos de alto riesgo y el craving en pacientes consumidores de PBC.

$\mathbf{H}_{1}$ : Existe relación entre los estímulos internos de alto riesgo y el craving en pacientes consumidores de PBC.

Nivel de significancia: Error tipo I, $\alpha=0,05$ (5\%). Esto es, el error que se cometería al rechazar la Ho, siendo esta verdadera.

Prueba Estadística: Prueba no paramétrica Chi cuadrado. Decisión Estadística: El Chi cuadrado observado es $\mathrm{Ji}^{2}=10,485$ con $\mathrm{gl}=4$, al cual le está asociado un valor de probabilidad, $P=0,033$. Puesto que este valor $P$ es menor o igual que $\alpha$, se concluye en rechazar la Ho. Es decir existe relación entre los estímulos internos de alto riesgo y el craving en pacientes consumidores de PBC.

\section{DISCUSIÓN}

Con respecto a la tabla 1 , en relación con las creencias nucleares y actitud sobre el craving en pacientes consumidores de PBC; el $30 \%$ es indiferente, mientras que el $18 \%$ están de acuerdo a sí mismo, el $12 \%$ se muestra en desacuerdo.

Las frecuencias observadas se sometieron a la prueba de hipótesis, lo que dio como resultado que exista relación entre las creencias nucleares y el craving en pacientes consumidores de PBC, el cual demuestra que la intencionalidad presente o futura en la conducta de consumir PBC tiene una direccionalidad positiva.

Al respecto Beck A., et al., (1999) plantean que las creencias adictivas parece que provienen sobre todo de una combinación de las creencias nucleares (a veces llamados esquemas nucleares). El primer grupo de creencias nucleares disfuncionales tiene que ver con la supervivencia personal, el logro o a motivación por conseguir determinadas cosas, la libertad y la autonomía.

Dependiendo de la naturaleza precisa de la vulnerabilidad del paciente, la creencia nuclear que se expresa puede tener un contenido como el siguiente: Estoy desprotegido, atrapado, derrotado, soy inferior, débil, inepto, no sirvo para nada o soy un fracaso. El segundo grupo de creencias disfuncionales tiene que ver con la vinculación con otros individuos o con un grupo. Esta clase de creencias se relaciona con los queridos o aceptados que son.

Las distintas permutaciones de estas creencias centrales o nucleares pueden ser de la siguiente forma: No soy querido ni deseado, soy indeseable y repulsivo, me rechazan, soy diferente y tengo defectos socialmente hablando. Estas creencias centrales predisponen una sensibilidad específica o vulnerabilidad: cuando aparecen algunas circunstancias (p. ej., el rechazo social) que son adecuadas para dicha creencia central, disparan la aparición de la misma (p. ej., tengo defectos) y producen la angustia.

Quiere decir, que nuestros datos, indican que las creencias nucleares activan el craving en los pacientes consumidores de PBC.

Con respecto a la tabla 2 , en relación a las creencias adictivas y la actitud sobre el craving en pacientes consumidores de PBC; el $24 \%$ es indiferente, mientras que el $20 \%$ están de acuerdo y así mismo el $10 \%$ se muestran en desacuerdo.

Estas frecuencias observadas se sometieron a la prueba de hipótesis, lo que dio como resultado que existe relación entre las creencias adictivas y el craving en pacientes consumidores de PBC, nuestros resultados 
coinciden con el informe que presentan Beck A., et al., (1999) que las creencias adictivas deben ser consideradas como un conjunto de ideas centradas alrededor de la búsqueda de placer, resolución de problemas, alivio y escape. ["Entre las ideas adictivas están a) la creencia de que uno necesita la sustancia si tiene que mantener el equilibrio psicológico y emocional, b) la expectativa de que se encontrará placer y excitación utilizándola, c) la expectativa de que la sustancia mejorará el funcionamiento social e intelectual, d) la creencia de que la droga energetizará al individuo y le dará más fuerza y poder, e) la expectativa de que la droga calmará el dolor, f) el supuesto de que la droga aliviará el aburrimiento, la ansiedad, la tensión y la depresión, g) la convicción de que a menos que se haga algo para satisfacer el craving o para neutralizar la angustia, ésta continuará indefinidamente $\mathrm{y}$, con toda probabilidad empeorará].

En la tabla 3 , en relación a las creencias anticipadoras y la actitud sobre el craving en pacientes consumidores de PBC; el $22 \%$ es indiferente, el $10 \%$ se muestran en desacuerdo y así mismo el $14 \%$ refieren estar en desacuerdo sobre el craving en consumo de PBC, pero se muestran de acuerdo con las creencias anticipadoras.

Estas frecuencias observadas se sometieron a la prueba de hipótesis, lo que dio como resultado que existe relación entre las creencias anticipadoras y el craving en pacientes consumidores de PBC.

Al respecto estos resultados coinciden con los hallazgos de BeckA., et al., (1999) que manifiestan que hay una secuencia de creencias en los consumidores de drogas, las primeras de la secuencia son las creencias anticipadoras. Al principio toman formas parecidas a: Será divertido hacer esto... Está bien probarlo ocasionalmente.

A medida que el paciente encuentra más satisfacción utilizándolo, la persona desarrolla creencias románticas que predicen la satisfacción o el escape: Tendré alrededor de una hora de pura satisfacción... Me sentiré menos triste y/o cansado ... será una forma dulce de olvidar. Algunas creencias son predictivas del incremento de eficacia o de socialización: Lo haré mejor... seré más divertido y se me aceptará mejor en el grupo.

A medida que la persona empieza a confiar en la droga para contrarrestar sus sentimientos de angustia, desarrollará creencias asociadas con el alivio, tales como: Necesito cocaína para poder funcionar... No puedo estar más sin ella... Me sentiré de nuevo bien si utilizo... Necesito la droga... No puedo controlar mi craving... Debo conseguirla o me desmoronaré. Uno de los aspectos que hay que notar es el tono imperativo de estas creencias: Necesito fumar para conseguir pasar el día. La activación de estas creencias conduce al craving.

Así mismo los resultados de la tabla 4, en relación a las creencias permisivas y actitud sobre el craving en pacientes consumidores de PBC; el $28 \%$ es indiferente, el $20 \%$ están de acuerdo con las creencias permisivas pero se muestran indiferentes (10\%) y en desacuerdo (10\%) respectivamente para el craving en consumo de PBC, por otro lado el $26 \%$ son indiferentes con las creencias permisivas pero refieren estar de acuerdo con el craving en consumo de PBC.

Estas frecuencias observadas se sometieron a la prueba de hipótesis, lo que resultó que existe relación entre las creencias permisivas y el craving en pacientes consumidores de PBC.

Este resultado coincide con el planteamiento en la teoría y terapia de la adicción que afirman que las personas con predisposición a las adicciones tienen algunos conflictos por su uso (p. ej., médicos, económicos, sociales o consecuencias legales de la utilización de las drogas), normalmente desarrollan una creencia facilitadora o permisiva, como: Me lo merezco o está bien, podré manejarlo... como me encuentro triste, está bien que lo use... No hay nada más que me funcione bien; está es la única cosa buena en mi vida.

En la tabla 5, en relación a los estímulos externos de alto riesgo y actitud sobre el craving en pacientes consumidores de PBC; el $22 \%$ se muestra en desacuerdo e indiferentes y así mismo el $20 \%$ están de acuerdo. Estas frecuencias observadas se sometieron a la prueba de hipótesis, lo que dio como resultado que existe relación entre los estímulos externos de alto riesgo y el craving en pacientes consumidores de PBC. También estos datos los corrobora Marlatt y Gordon (1985) a través del Modelo Cognitivo - Conductual, el cual plantea que la persona adicta a la cocaína es vulnerable a los estímulos de alto riesgo (EAR). Dicho modelo subraya la existencia de factores tanto personales como una pérdida de control ante una situación de alto riesgo.

Con respecto a los estímulos externos de alto riesgo incluye, lugares, personas y cosas que están relacionadas de alguna forma con la utilización de las drogas (Shulman, 1989). Cuando los individuos se enfrentan a estas situaciones de alto riesgo deben responder con conductas de afrontamiento.

Aquellos que tienen respuestas de afrontamiento afectivas, desarrollan mayor autoeficacia y como consecuencia existe menos probabilidad de recaída. Aquellos se tienen relativamente menor número de respuestas de afrontamiento o ninguna, experimentarían una disminución en su autoeficacia y un aumento de las expectativas sobre los efectos positivos que produciría la droga, seguido de un desliz, es decir, empieza de nuevo el consumo de droga.

Tal y como vimos antes, el craving aparece en una situación específica y parece que se active como una reacción refleja al estímulo.

Sin embargo, la situación no "causa" directamente el craving, se encuentra la creencia relacionada con la droga que se activa por la situación y por un pensamiento automático derivado de esta creencia. La secuencia evoluciona hacia el craving, las creencias facilitadoras, hasta un plan de acción para obtener la droga y utilizarla al final.

O'Brien (1992) requiere que la frecuencia ocurre tan rápidamente que normalmente se suele considerar como un reflejo condicionado. En particular, el pensamiento automático parece ser instantáneo y sólo se puede apreciar si el paciente aprende a poner atención a las cadenas de acontecimientos.

En la tabla 6, en relación a los estímulos internos de alto riesgo y actitud sobre el craving en pacientes consumidores de PBC; el $20 \%$ están de acuerdo y el $18 \%$ se muestran indiferentes. Estas frecuencias observadas se sometieron a la prueba de hipótesis, lo que dio como resultado que existe relación entre los estímulos internos de alto riesgo y el craving en pacientes consumidores de PBC. Al respecto Mackay y otros (1991) plantean que los estímulos internos incluyen factores emocionales y físicos como la depresión, la soledad, el aburrimiento, la ira, la frustración y el dolor físico.

Las personas que sufren adicciones al alcohol y a las drogas encuentran inevitablemente estímulos de alto riesgo. Esto es muy evidente cuando se considera el hecho de que todas las personas experimentamos en determinados momentos algo de tristeza, ansiedad, nerviosismo, ira o frustración (todos ellos estímulos de alto riesgo interno, EAR). 
La identificación de estos EAR internos y externos es un componente extremadamente importante del proceso de prevención de recaídas, es decir, tener tendencia a exponerse a situaciones de alto riesgo y estar poco preparado para manejar sus pensamientos, sentimientos, craving y acciones resultantes.

\section{REFERENCIAS BIBLIOGRÁFICAS}

Aspillaga, M. (2011). Creencias irracionales y estilo atribucional en un grupo de abusadores de marihuana. Tesis de pregrado. Perú: Pontificia Universidad Católica del Perú.

Beck, A.[et. al.]. (2013). Terapia cognitiva de las drogodependencias. Barcelona: Paidós.

Blasco, J. y Col. (2008). Atención y Craving o ganas compulsivas. Avances en su conceptualización y su implicación en la prevención de recaídas. En Revista Adicciones, Vol. 20, № 4, pp. 365-376.

Bocanegra, C. (1990). Las creencias irracionales: Un estudio comparativo entre un grupo de sujetos consumidores de $P B C$ y un grupo de sujetos no consumidores de drogas. Un enfoque racional emotivo. Tesis de Pregrado. Perú: Universidad Nacional de San Marcos.

Brailowky, S. (2006). Las sustancias de los sueños. Neuropsicofarmacología. México: IEPSA.

Caroll, K. (2001). Un enfoque cognitivo conductual: el tratamiento de la adicción a la cocaína. Madrid: Fundación de Ayuda contra la Drogadicción y Citran.

Cartagena, G. (2000). Niveles de conducta emocional inadaptada en dependientes a PBC en comparación con no consumidores. Tesis de pregrado. Perú: Universidad Peruana Cayetano Heredia.

Cedro (2011). El problema de las drogas en el Perú. Lima: Cedro.

Cedro (2013). Epidemiología en población urbana peruana 2013. Lima: Cedro.

Chesa, D. (2007). El craving, un componente esencial en la abstinencia. Girona: Hospital Psiquiátric de Salt. Institut d'Assistencia Sanitaria.

Conyer, R. (2001). La cocaína, origen, botánica e historia. En las adicciones: dimensión, impacto y perspectivas. México: Manual Moderno, México, p. 219.

Devida (2012). Estrategia nacional de lucha contra las drogas 2012 - 2016. Lima: Comisión Nacional para el Desarrollo y Vida sin drogas.

Devida (2014). Información básica en reducción de la demanda de drogas. Lima: Comisión Nacional para el Desarrollo y Vida Sin drogas.

Devida (2005). Revista Peruana de Drogodependencias. Análisis e investigación. Vol 3 № 1. Lima: Comisión Nacional para el Desarrollo y Vida Sin Drogas.

Espada, J. y Col. (2010). Terapia psicológica. Casos prácticos. Madrid: Pirámide.

Ferreira, A. (2000). Estudio sobre el soporte social y la interacción familiar y su influencia en la conducta adictiva a la PBC en un grupo de jóvenes de nivel socioeconómico medio. En Revista de la Facultad de Psicología de la Universidad Nacional Mayor de San Marcos, pp. $141-156$

Gómez, A. y Conde, A. (2004). Craving y alcoholismo. En: Revista Médica Clínica V(6), No 123, . pp. 34-35.

Gonzáles, I. (2009). Estrategias cognitivo - conductuales para el manejo del craving. En: Revista de Toxicomanías $\mathrm{N}^{\circ} 57$.

Guardia, J. y Col. (2004). Estudio de la validación de la escala multidimensional de craving de alcohol. Barcelona: Unidad de conductas adictivas. Servicio de Psiquiatría. Hospital de la Santa Creu i Sant Pau. Disponible en http://www.elsevier.es
Hernández, R. y Col. (2010). Metodología de la Investigación. México D. F.: McGraw - Hill Interamericana.

INPE - Devida (2013). Programación de intervención R.E.T.O. Lima: Programa Ambulatorio en el Medio Libre del Instituto Nacional Penitenciario y la Comisión Nacional para el Desarrollo y Vida Sin Drogas.

Iraurgi, I. y Corcuera, N. (2008). Craving: Concepto, medición y terapéutica. En: Revista Norte de Salud Mental № 32 , presentado en: II Simposium sobre alcoholismo y ludopatía. Gobierno Vasco. pp. 9-22.

Iraurgi, I., y Col. (2009). Fiabilidad y validez de la versión abreviada en español del Cocaine Craving Questionnaire - Now (CCQ - N - 10). En: Revista Adicciones, Vol. 21, № 3. España. pp. 195-202.

López, A. y Becoña, E. (2006). El Craving en personas dependientes de la cocaína. En: Revista Anales de Psicología, Vol 22, № 2 de la Universidad de Santiago de Compostela de España. pp. 205-211.

Martínez, J. y Verdejo, A. (2011). Creencias básicas adictivas y craving. En: Revista Adicciones, Vol. 23, № 1. España. pp. 339-352.

Martínez, J. y Verdejo, A. (2012). Evolución de las creencias nucleares relacionadas con la adicción en drogodependientes con y sin trastornos de personalidad. En: Revista Adicciones, Vol. 24, № 3. España. pp. $229-$ 238.

Miraval, E. (2003). Farmacodependencia y Alcoholismo. Perú: Edición Impresiones Horizonte.

Muñoz, M. Mecanismos motivacionales del craving: ansia por el tabaco. Tesis Doctoral. Facultad de Psicología. Universidad de Granada.

Navarro, R. y Col. (2001). Perfil de un grupo de pacientes adictos recuperados. En: Revista Latinoamericana de Psiquiatría, V(1) No 1, pp. 30-35.

NIDA. (2001). Principios de tratamiento para la drogadicción: Una guía basada en investigaciones. Disponible en: http://www.nida.nih.gov/PODAT/Spanish/PODATIndex. html

OPD (2009). Análisis de resultados de la red de información de demanda de tratamiento por abuso o dependencia de sustancias psicoactivas 2005-2007. Lima: Observatorio Peruano de Drogas y Comisión Nacional para el Desarrollo y Vida Sin Drogas.

Rodríguez, A. (2012). Afrontamiento y soporte social en un grupo de adictos de un Hospital Psiquiátrico de Lima. Tesis de Pregrado. Perú: Pontificia Universidad Católica del Perú.

Rojas, M. (2006). Conceptos básicos y profundización del consejo psicológico y terapia motivacional en drogodependencias. Un Enfoque Cognitivo Conductual y Humanista. Lima: Cedro.

Rojas, M. (2007). Creencias irracionales en mujeres y varones consumidores de cocaína. Una mirada desde el enfoque cognitivo. Lima: Escuela de Post-Grado. Víctor Alzamora Castro de la Universidad Peruana Cayetano Heredia.

Rubio, M. (2009). Papel del sistema cannabionide endógeno en el alcoholismo: Implicaciones fisiológicas y terapéuticas. Tesis Doctoral. España: Universidad Complutense de Madrid.

Vela, O. (1990). Creencias y concepciones irracionales en el consumidor de PBC. Tesis de Pregrado. Perú: Universidad Ricardo Palma.

Vigo, M. (1999). Creencias irracionales en varones y mujeres dependientes de cocaína. Tesis de pregrado. Perú: Pontificia Universidad Católica del Perú.

Zapata, M. y Segura, A. (2007). Factores predictivos para el resultado de tratamiento en pacientes farmacodependientes. En Revista Peruana de Drogodependencias, 5(1). pp. 159-185. 